\title{
Application and Design of New Media in Museum Exhibition and Display
}

\author{
Jinxiu Wang \\ Leshan Teachers College \\ hunter2011@foxmail.com
}

Keywords: New media technology; Exhibition and display of museum; Traditional concepts

\begin{abstract}
In recent years, new media spring up suddenly compared with traditional media. However, the application of digital technology in exhibition and display of museum not only has great influence on the development of exhibition but also brings much attention on digital exhibition and display technology. New media can effectively transmit and display the exhibited information of museum and the information it displayed together with traditional media. New media and traditional media combine organically and supplementary to each other in the exhibition design of museum. We should emphasize the great value of new media in the application and design of museum exhibition and design of museum; meanwhile, it should break the restraints of tradition thinking patterns and strengthen the application study of new media technology in museum exhibition and display so as to actively promote the influence of new media in the development of museum.
\end{abstract}

\section{Introduction}

Nowadays, with the rapid development of science and technology, new media is widely used in various industries and it promotes the exhibition and display of museum in China into a new stage. The digital exhibition of museum has increased rapidly with the new media technology. Therefore, increasingly strengthening and study the influence of new media technology in museum exhibition and display is of great importance in promoting the construction and development of museum career in China. However, museum is to exhibit the real collections so as to exhibit and transmit it in traditional sense. It is exhibited by cultural relics and collections with the help of words description, pictures and some painting methods to communicate with people so as to realize the function of museum display and exhibition. However, this static method in traditional concept cannot catch up with the era. Therefore, study on the application and design of new media technology in the museum exhibition and display is of great importance.

\section{Interpretation of new media technology concept}

New media technology is a great evolution in the rapid developed high technology field. It brings computer image, cyber reality and artificial life into the new culture of the new era. The footsteps of human civilization now enter a visual-oriented new culture era. New media is relative to traditional media; new media is born on the basis of traditional media and is beneficial from the high technology and gradually improved in social practice. New media is based on digital information technology with the features of interactive transmission. Traditional media, such as newspaper, broadcast, television, magazines etc., are gradually replaced by digital newspaper, digital television and touch media. New media technology developed under Internet technology plays its information service function and also has innate technical advantages. Compared with traditional media, the new of new media lies in the innovation in technology, forms and theories. It provides information; meanwhile it can realize the equal communication between communicator and audience or between audiences.

With the constant development of new media, its features also receive wide attention. The feature of new media here is compared with traditional media. Firstly, new media has a disarming power which can eliminate the boundary between media and media, country and country as well as industry and industry. Secondly, it has interactivity and can cross time and space as well as the 
transmission between points and points. Thirdly, new media also constantly develops. New media is constantly advanced and it would not terminate at certain state as well as a constantly advanced media. And then, new media can integrate information and it can integrate of the diverse and complex demands of audiences. In the development of museum, traditional media and new media all play their different functions and mutually play the role of information transmission.

\section{Concrete effect of new media in the application and display of museum exhibition and display}

The exhibition of collections by museum is, in fact, the museum handling of collections and displays the huge information in the exhibits. People can study the collections by exhibition so as to dig out the historical information of collections as well as its historical values and significant of era, which is the everlasting aim of museum exhibition and display. However, new media plays great role in the design of museum exhibition and display, which can realize the everlasting aim of museum exhibition and display with rapid and effective as well as scientific methods. New media can interact with various sense organs (such as audition and visual as well as sense of touch and smell etc) so as to arouse the interests of audiences in exhibition and display, which can effectively interpret history and greatly improve the activeness for exploration of knowledge and cultivate the sentiment of audiences for science.

Influence of new media in the design of museum exhibition and display. Traditional museum is often exhibited by displaying the collections, which is a static display method. Traditional display methods cannot satisfy the demands of audiences. Museum is an important learning place for learning traditional cultural knowledge and it gets more close to people's life. And its operation concept also gradually transformed into human-oriented. Therefore, exhibition also displays the redundant information to the public. From both the exhibition content and the comprehensive exhibited design, new media plays irreplaceable role. Firstly, new media digital technology can enrich the exhibition language of museum and can be designed into different exhibition methods can reach better display effect. Secondly, exhibition space also changed with the development of digital technology.

On the basis of traditional "physical space", it comes new cyber space to display the space and it can expand the space range of museum exhibition. It can even restore the real story by digital technology so that the audiences can better absorb and understand traditional culture knowledge. Besides, museum exhibition and display can interpret the cultural relics and the bearing history information with a new method and from new perspectives. It can tell the deeper history story with digital method and skillfully apply digital methods so as to deepen the theory of exhibition and display so as to touch the public. Finally, during the application and design of exhibition, it can vividly present the limited historical materials to the public by a series of media technology and public can also have increasing information to choose. Publics can actively participate at the scene by technology so as to vividly reflect the historical truth.

Museum exhibition and concrete application of new media technology. Museums in foreign country apply new media early. At the end of previous century, there are examples of bringing in new technology in the museum exhibition and display so as to build up different websites and social platforms for groups at different age and with different groups. Meanwhile, it develops many mobile equipment and procedures so that public can participate in it. The application of new media in museum starts late compared with foreign countries. Many museums start to pay attention to new media in recent years. By mobile phone, iPads and other media platform, it can apply digital methods to enrich the exhibition contents and forms so that more and more people can share the museum information and resources without the limitations of boundary. As for ordinary audiences, they cannot fully understand their inner meanings of exhibition. However, with the help of new media, it can perfectly store the huge information of the collections; people can understand the materials, functions, historical information, cultural information, connotation information and other information connected with other things so as to maximum the understanding for exhibited collections. As for the application and design of museum exhibition and display, the concrete application of new media technology mainly consists of the following aspects: 
Firstly, application of screen projection system in museum exhibition. Static exhibition is the exhibition method in traditional sense. The elements such as television, animation and sound, have great effect on the display effects. It can display the contents with many forms and many layers. The setting of screen projection system can play the content dynamically for museum. The combination of sound and animation increases the advertising strength. By digital handling technology, people feel as if they are at the scene so as to concretize the abstract historical information and contrast the exhibition effect by rendering the atmosphere.

Secondly, the application of touch technology. It has the advantages of small volume, large contents, rich information presentation forms, strong interaction and convenient operation. The design of touch content of museum can edit the museum information and audiences can understand or selected their interested information by touch screen so as to satisfy the demands of audiences for information and make the operation more convenient and easy.

Thirdly, the application of spectra vision by taking new media technology as platform. The most important of the museum exhibition is cultural relics and collections. Affected by the limitations of space, audiences cannot appreciate the precious cultural relics which cannot move. The spectra vision system in new media technology, as a new exhibition system, can realize the digital virtual display from all aspects and display more information with little space which is not limited by time or space.

New media and design of museum display. The application of new media in exhibition display mainly achieves better effect by assisting the exhibition. New media can integrate information and realize the display of various complex information by combining images, words, sound etc. And meanwhile, it can cater to the demands of receiving information by various senses, which is a new media and new forms exhibition. Meanwhile, the integration of cross media can realize the intricate communication of media. It can flexibly select proper means and methods based on the concrete demands of exhibition to realize the transmission mode of exhibition and audiences so to maximize the exhibition experience of museum.

During the application of new media technology in the museum display, there is the fusion of new media concept which is easy to be ignored. The new media has some breakthrough innovations in the transmission concept compared with traditional media. The most remarkable application in museum exhibition is the conceptual art of new media. During exhibition of cultural relics and collection, the concept in the exhibition would far more than their study value, which can attract more interests from the audiences. The expression of this concept is new and belongs to new media. In application of museum display design, new media is the tool and equipment for communication and also plays important role in the organization of museum, the construction of knowledge framework and the attention of public.

\section{New media in the development of museum}

Application of new media technology is not surprising in the design of museum exhibition in China. At current stage, however, the bringing in of this new technology is in its early stage and people have to pay attention to the problems existing in the development. Affected by traditional concept, though new media has booming development, the interpretation and understanding for its features also have some misunderstanding and blindness, including some people in museum. There are two extreme understanding for new media technology, including the blind bringing in of new media technology and avoiding bringing in new media technology to reduce the attention on traditional exhibition.

Besides, the bringing of new media technology should base on the concrete conditions of museum exhibition. The selection of new media technology should be regarded as a technical method for museum exhibition instead of replacing the leading role of cultural relic's exhibition. The application and design of new media in museum exhibition gradually transform the operation concept of museum. From the leading place of traditional media to the bringing of new media technology, museum breaks the limitations of traditional concepts and eliminates the distance between itself of the audience so as to display the static collections to the audiences with flexible 
and changeable technology. Audience can select their own interested or beneficial information with new media technology to realize good transmission effect. During this process, concept is quite important. Traditional concept and changeable environment would affect the development of new media technology.

Nowadays, the development of digital development is inevitable. New media technology has involved in many links of museum work. New media technology plays its active role and the existing problems should also arouse our attention. The first is the high investment on exhibition for the misuse of various new media technology and pursuit of high invest and high produce; the second is to cater to the market and absorb eyeball of people, exaggerate or empty the historical values of some cultural relics. The third is to pursue economic benefits in the exhibition of museum and ignore its educational function, lack of individualized design etc.

\section{Conclusion}

In a word, with the constant development of digital technology, new media has special study significance as the supplementary to the application and design of museum exhibition. It has certain importance from the exhibition forms and exhibition effects. However, it does not mean that museums can randomly bring in new media technology for they have to select the new media technology to improve exhibition effect based on concrete situation. Breaking the restrictions of traditional concept and audiences-oriented can improve the exhibition connotation, constantly innovate thinking so as to rationalize and widen the application of new media in the museum exhibition.

\section{References}

[1] L. Ren. Analysis on new media exhibition technology of museum---- Starting from combination of Mountain and river, special exhibition of Huang Gongwang and Dwelling in the Fuchun Mountains. Read and Write Periodical, 2012, 23(05):75-76.

[2] G. Chen. New media and museum information transmission. Chinese Museum, 2012, 15(01): 25-28.

[3] Z.X. Song. Application of new media--New trends of museum. Stories of Relics, 2014, 13(01): 47-51.

[4] L.Z. Liang. Status quo and its transmission effect study for municipal museum in Jiang Su province. Nanjing University of the Arts, 2013

[5] C. Hu. Application of new media exhibition in history museum for museum experience. Hunan Normal University, 2013 